\title{
COM \\ A cybernetic dream: how a crisis in social sciences leads us to a Communication for Innovation-Laboratory
}

\section{Maarten C.A. van der Sanden and Steven M. Flipse}

\begin{abstract}
After the first paradigm shift from the deficit model to two-way communication, the field of science communication is in need of a second paradigm shift. This second shift sees communication as an inherently distributed element in the socio-technical system of science and technology development. Science communication is understood both from a systems perspective and its consecutive parts, in order to get a grip on the complex and dynamic reality of science, technology development and innovation in which scientists, industrial and governmental partners and the lay public collaborate. This essay reflects on the under-development of system thinking in science communication and the need to fix this. Legitimation for the second paradigm shift is found in the 'crisis in social sciences' that has led to a revival of system theory to balance the deterministic thinking in our grounding discipline. This essay concludes with the idea of a 'Communication for Innovation-Lab' as an experimental setting in which whole/part thinking in science communication can be shaped according to this second paradigm shift, forming seed crystals for future developments.
\end{abstract}

Keywords

Second paradigm shift, Social systems theory, Communication for Innovation Lab

Introduction:

from Mode-2 to systems thinking
Professionals in their daily practice of science communication, including e.g. scientists, experts in communication, innovation, education and policy making, take decisions in ill-defined complex situations of science and technology development in which they use their knowledge, experience, creativity and intuition [van der Sanden et al., 2013; Abma, 2011]. They need to deal with current practices of science and technology development, which are often considered to have Mode-2 science [Nowotny, Scott and Gibbons, 2001; Kropp and Blok, 2011] characteristics. In Mode- 2 science, universities lose their monopoly on knowledge production, as research is conducted within ad hoc interdisciplinary networks, organized in close collaboration with non-academic partners often within the contexts of the application of the research, and in response to social, economic, and political considerations and expectations about the practice of science and technology [Kropp and Blok, 2011].

The first paradigm shift in science communication from one-way to two-way communication [Wynne, 2001] was induced by this idea of Mode-2 science. Many 
authors in science communication wrote about dialogue and debate, and continue to do so. The goal of such public engagement (or even public participation) can be to explore with the public what people want from technology in the future, with the aspiration that science and society can work together to shape that future [cf. Jones, 2011]. However, if one wants to understand public engagement or participation in this social realm of Mode-2 science, the collaboration between scientists and their scientific partners and its dynamic properties should also be taken into account. Namely, as we can learn from corporate communication [van Riel and Fombrun, 2007], the identity of scientists and their values and needs as partners of co-creation on the level of public engagement and participation, is partly based on the values and needs of scientists on the level of collaboration with their scientific peers [van der Sanden and Osseweijer, 2011]. Scientists, industrial researchers, university and governmental policy makers on science and technology and the public, co-exist, collaborate and co-create on various levels from various roles in the socio-technical system of science and technology development.

To make the argument of a second paradigm shift that entails system thinking in science communication, we will first introduce this idea of the second shift in light of earlier work. We then introduce the concept of system thinking in relation to science communication. The essay moves on to focus on the crisis in social sciences, and the possible 'way out' by focusing on system thinking to balance the deterministic thinking. This is followed by a brief outline of social systems which shows that this cybernetic view is actually not new to the social sciences. And finally we conclude with a cybernetic dream of a Communication for Innovation-Lab that embodies whole/part thinking.

The second paradigm shift
In an earlier book chapter [van der Sanden and Osseweijer, 2011] on effectively embedding science communication in academia, we introduced the concept of a second paradigm shift in science communication in which scientists are encouraged by their professional academic institutes to strengthen and streamline their efforts in science communication and to incorporate science communication activities into organisational policy. We proposed this could be done in 3 steps:

1. Linking science communication with university policies and strategies;

2. Aligning motivation with incentives;

3. Providing a learning culture for coordinated science communication.

By 2035, as we wrote: "this second paradigm shift will have completely changed science communication. It is now established as an integrated and valued part of the research organisation's overall strategy." Along this line of thought, Scheufele [2014] recently wrote about the urgent need to incorporate political science: "[...] Science communication will therefore have to draw much more than in the past on theorizing and empirical work in political communication, public opinion research and related fields. This reliance on empirical social science will be crucial to understand and participate in the processes that determine how science gets communicated and debated in real-world settings."

To take this further, next to science education, social studies of science and communication studies [Mulder, Longnecker and Davies, 2008], also e.g. marketing 
becomes more important to real-world settings in relation to funding, the international (online) education context, and international scientific labour market, since every university would like to have top research executed by top researchers [van der Sanden and Osseweijer, 2011].

Moving to systems thinking
As the process of science becomes more dependent on collaboration in multidisciplinary networks in which new (innovative) ideas are co-developed, scientific development is seen as a more complex 'socio-technical system'. A system in which scientists, industry, government and the public co-exist, collaborate and co-create, while their goals, values and needs are intertwined. In such collaborations communication plays a crucial role to overcome potential social dilemmas [Tromp, 2013]), e.g. dilemmas in cooperation if one wants to reach goal congruence in teams [Pennington, 2011] or social learning between stakeholders [Wenger, 2000]. Thereby, the role of the science communicator can change to that of a broker [Meyer, 2010] or coach [van der Sanden and Osseweijer, 2011].

The science communicator becomes someone who is interacting in a network and understands the dynamics of collaboration between partners within that network [Flipse and van der Sanden, 2014]. If we consider the collaboration between scientists and their shared values and goal congruence, we can also better understand part of the dynamics of upstream engagement in which actors cooperate. Here, science communication can be a lubricant or glue that runs and connects a system of distributed elements of considerations, values and goals of scientists, technicians, partners, industry, government and lay audience, in which all parts are connected. Currently we mostly investigate parts of this information on various systems levels, yet this second paradigm shift stimulates thinking about both the whole system and its consecutive parts. Such 'whole/part' thinking then should lead to more inclusive science communication innovations that work in the daily complex and dynamic practice of science communication. The second paradigm shift then concerns the following: science communication becomes a distributed system-element in a socio-technical system of science \& technology development in which each actor has a communicative role, function and tasks, that needs to be stimulated, supported and trained, for real world complex and dynamic multidisciplinary collaboration in all stages of science and innovation.

Such system thinking in science communication entails that neither collaboration, nor participation, engagement and understanding are exclusive. They all co-exist in the socio-technical system of scientific and technical development and innovation. Moreover, this science communicative system can only be understood from the aforementioned multidisciplinary angle in which empirical social science plays an important role. Interestingly, during the PCST-13 conference in Brazil, Martin Bauer brought out an essay-competition for Public Understanding of Science about the inevitable recurrence of the deficit model. Concerning the system idea as described above this recurrence is not strange or undesirable, since straightforward "teaching" or "science education" for public understanding is still needed. It simply has never disappeared from the overall system of science communication. As such, engagement with science and straightforward understanding of science do perfectly co-exist on different levels and stages of the social system of science communication. 
Crisis in the social sciences

This section is mainly based on a book written by Ruud Abma [2011]: Beyond the boundaries of disciplines. Localisation of social sciences. In this book Abma writes about social science in crisis concerning its societal relevance, e.g. in how to make such relevance clear and tangible for policy makers. As he writes that, social scientists deal with a difference between their nomothetic theories at one side and procedures and practice in the real world at the other. Although there are social engineers who support citizens and policymakers to make the complex social world manageable by interpretation frames and means which forms evidence-based practices, modern sociology is still built on logical positivism [cf. Steinmetz, 2007].

This way of thinking entails understanding behaviour on the level of individuals, and by rules of transformation connecting the individual level with the group level. However, frequently social scientific interventions are not closely enough related to the daily practice of experts, preventing a good fit between interventions and functionality in practice. This is based in part on a lack of insight into how experts take decisions using (social scientific) knowledge. That is what Abma calls a lack of 'naturalistic decision making'. Practice is much more than the application of theory. Moreover, professionals need to participate in research to achieve practice-based evidence [Abma, 2011] next to evidence-based practice.

So social sciences are at the crossroads between being a more instrumental natural science or part of more reflexive humanities ${ }^{1}$ [Penders, Verbakel and Nelis, 2009]. Kropp and Blok [2011] state: "Given that most Mode-2 discussion takes place in reference to natural and technical sciences, this also implies an urgent need for more studies of transformed dynamics of knowledge production in the humanities and the social sciences." Complexity and systems theory was and remains a mediating principle. This crisis in sociology is all about the systems level of social issues (as Abma argues), and researchers and professionals should use whole/part thinking in finding solutions that can be translated to practice. We argue below that they frequently already do so in the realm of science communication.

Yet, is this a revolution, as critics of this 'crisis' ask? According to Eliasmith [2012] it is not. And the 'standard' cognitive science is adequate (or even more appropriate) for understanding the Complex Systems Approach (CSA) provided examples. He writes: "In conclusion, I would like to again emphasize that I take none of my considerations here to suggest, in any way, that dynamical, non-linear, interaction dependent models are undesirable. Quite the opposite, in fact. [...] The point simply is that calls for revolution in cognitive science are vastly overstated. Surely cognitive science has many challenges ahead of it, but it is not in a state of crisis as proponents of CSA would have us believe."

In science communication many professionals and researchers recognize the same gap between theory and practice, and thereby implicitly the need for system thinking and its whole/part thinking. Not to say that science communication is in a theoretical crisis, but in a strong need to make system thinking explicit. And indeed as aforementioned the second paradigm shift is not about excluding the first one, or deficit thinking, or deterministic thinking, but rather about including the first shift and considering science communication as a system element in the social system of science and technology development.

\footnotetext{
${ }^{1}$ A discussion that analogously has also been raised, compares STS as a movement, with STS as a field of study [Penders, Verbakel and Nelis, 2009].
} 
System thinking

as a cybernetic

tradition for

science

communication:

boundaries,

social learning

and social

entropy
Actually the system idea has an early predecessor, namely the 'cybernetic tradition' in communication science. As Littlejohn and Foss [2008] write: "Within cybernetics communication is understood as a system of parts, or variables, that influence one another, shape and control the character of the overall system, and, like any organism, achieve both balance and change." For example, research project proposals, as an outcome of collaboration between scientists and other stakeholders, are framed in such a way through collaboration that some research aspects are emphasized and others are not. Or in meetings of scientists with governmental policy makers, certain concepts prevail. Boundaries between what is included and what is not occur and disappear. Bailey [1994] writes: "Boundaries become structures which not only define spatial area but also constrain social interactions. Thus boundaries are in a dialectical relation to process (social interaction) just as all structure is."

One of the scientists in the group may act as a boundary spanner by searching for consensus or contribute to the discussion with another creative angle. This may even change the social structure of the collaborative network over time. The social system theorists like Luhmann [1995] Luhmann describe this idea of boundaries as Wenger [2000] does in discussing social learning. Social learning can be seen as a dynamic process based on engagement, alignment and imagination when professionals collaborate. Wenger writes: "Their (organizations, authors) success depends on their ability to design themselves as social learning systems and also to participate in broader learning systems such as in industry, a region or a consortium". The latter could be a consortium of collaborating scientists.

Bonding and bridging between scientists, between scientists and industry or between scientists and the lay public, plays a role in the way in which actors interact (or do not ${ }^{2}$ ) within the group and interact with other groups [Burt, 2000]. The effort that all this takes is the energy brought up by the participants to keep the network together. This energy flow in social systems can be interpreted based on Social Entropy Theory (SET) as discussed by Bailey [1994]. SET says that every system wants to disintegrate. Luhmann [1995] and Bailey [1994] mention that in these social networks structures, procedures for social interaction occur and that communication energy is needed to keep the system from disintegration. The same holds for social systems in science communication [Sanden and Meijman, 2007]: without communication 'energy', the system falls apart. Moreover, such dynamic systems are characterized by self-regulation and control. Therefore, system theorists are not simply interested in the nature of the system and its functions but in how it manages to sustain and control itself over time [Littlejohn and Foss, 2008].

Furthermore, there is emergence. In his book on Social Emergence Sawyer [2005] tries to make a connection between parts and the whole system (holistic \& deterministic thinking). Sawyer also describes the various levels of emergence in which individuals, groups and society cooperate. He mentions stable emergent elements (group culture, group language, collective memory) and fluid emergent elements (structures of participation, role and status distribution). Differences in communication processes and means entail differences in emergence and outcomes of social systems [Abma, 2011]. In various disciplines, and also for social science, the idea of emergence is a leading principle instead of reductionism (ibid). In this idea, reductionism (logical positivism) and emergence co-exist. Whole/part

\footnotetext{
${ }^{2}$ Also silence in such networks plays a role, when contributors stay silent for team or goal sake [Verouden and van der Sanden, 2013].
} 
thinking comes much closer to reality and experts are actually doing all this implicitly on a daily basis. They are aware of the idea that the corporate university's identity is based on how its scientists behave in all kinds of collaborative settings, ranging from industrial or governmental collaboration to guest lectures on secondary schools for marketing purposes. So, if we understand how values of scientific research and its according theories prevail in meetings of scientists and at the cost of what amount of discussion, we also obtain more understanding of the 'emergent surprises' in how scientists behave in a public debate or in other outreach activities intended or a lay audience. These insights may lead to an improved science communication support of scientists, R\&D managers, governmental policy makers and the lay audience. Support that takes dynamic changes in communication roles and functions into account and empowers actors in the science communication system to become more reflexive and adaptive.

A cybernetic dream

How could we proceed with this movement toward the system at which practitioners and researchers in social science and science communication point? How to study the specific dynamic interrelation between communication elements in these socio-technical systems in which science and communication are inherently attached? How to deal with science communication that is not only important from a democratic perspective, or economical perspective, but is also needed to understand science and scientific development itself in for example innovation? How to deal with whole/part thinking in research and practice?

Communities of practice in science communication, in which practitioners and researchers collaborate, that originate at this very moment (we for example are partners in SciComLab ${ }^{3}$ ), attempt to combine evidence-based practice with practice-based evidence. These are typical collaborative networks that overarch deterministic theoretical outcomes of research and emergent practices of science communication. The participants together are a network of system thinkers that brings together the whole and parts of science communication.

To investigate and understand this whole/part thinking in more detail we would like to establish a real laboratory: the Communication for Innovation-Lab (CI-Lab). In this CI-Lab professionals collaborating for innovation do their daily work and make their decisions in real time, while being observed and supported by training and decision support tools developed by science communication researchers. For example, by simulating collaboration for innovation between scientists and university policy makers in the context of responsible research and innovation [Flipse et al., 2014].

In the middle of the lab there is a huge (digital) table around which practitioners (i.e. science communication professionals, scientists, governmental policy makes,

\footnotetext{
${ }^{3}$ SciComLab is a Dutch community of practice originated from the Dutch association for science communication professionals, SciCom.NL. The group of 10 professionals consists of science communication officers from universities, science journalists, commercial science communication professionals and science communication researchers. We currently work on: evaluation methods and tools for science communication that can be executed by the professionals themselves on a daily basis. At the same time we evaluate our collaboration process to learn from it for future collaborative innovations. The latest post (in Dutch): (https://scicomnl.wordpress.com/2014/06/01/scicomlab-nieuwecommissie-resultaat-workshop-wtc2030-1604-verslag.)
} 
lay audience, etc.) and researchers discuss the outcome of their decisions and the ideas for the next step in training and tooled support. This direct interaction between the CI-Lab participants and the possibilities for immediate trial and error, sheds light on the various levels of the social technical system and their dynamic interrelations and the inevitable bounded rationality of all its participants (i.e. humans beings have a partly view on complex problems) and how to cope with that in the real world by developing different science communication scenarios.

The decision support tools and serious games through which professionals in the CI-Lab can understand the possible dynamic interaction and act upon, contain the socio-psychological details based on observations, socio-psychological (system) theories and according surveys amongst users of the tool [van der Sanden et al., 2013]. Based on the CI-Lab table discussions tools are modified on a detailed level and prepared for reuse within the real world setting, the science communication system, in the CI-Lab. By doing this we follow Scheufele's idea of empirical social science for science communication, attached to Abma's [Abma, 2011] idea for system thinking in social sciences, that entails new technology such as multi-agent simulation and serious games.

This kind of lab-research attached to communities of practice helps to discuss with each other the parts, the whole and the emergent properties of science communication and how to deal with all this by developing various scenarios for the real practice of collaboration for science communication innovation.

So the second paradigm shift is all about considering science communication as a system in which whole/part thinking is a prerequisite for understanding the complexity and dynamics of science communication. And yes, system thinking (considering the whole and its parts at the same time) is not a panacea heading for perfect solutions that directly fit into the daily practice of science communication. System thinking should indeed not be overrated, as we perhaps did when we said that two-way communication is more important or effective than one-way [van der Sanden and Meijman, 2008; Trench, 2008].

Therefore the second paradigm shift we propose starts from the principle that science communication needs to be considered as a distributed system-element in socio-technical systems in which each actor from scientist to lay public needs to be stimulated, supported and trained to reach insights for real multidisciplinary and multiparty collaboration in research and practice.

The CI-Lab researches, stimulates, supports and trains its participants. It supports the idea of the reflexive practitioner [Schön, 1983] who applies innovation in his own complex and dynamic practice of e.g. responsible research and innovation [Stilgoe, Owen and Macnaghten, 2013]. The CI-Lab offers one view and insights of the cybernetic dream that constitutes the second paradigm shift in science communication and helps to plant these thoughts and let discussion take off as seed crystals for future developments. wetenschappen. Nijmegen, The Netherlands: Vantilt. 
Bailey, K.H. (1994). Sociology and the new systems theory: toward a theoretical synthesis. New York, U.S.A.: State University of New York Press.

Burt, R.S. (2000). 'The network structure of social capital'. Research in Organizational Behaviour 22, pp. 345-423.

Eliasmith, C. (2012). 'The complex systems approach: rhetoric of revolution'. Topics in Cognitive Science 4, pp. 72-77.

Flipse, S.M. and van der Sanden, M.C.A. (2014). 'A role for communication professionals in support of responsible innovation practices'. In: Proceedings PCST-13. (Bahia de Salvador, Brazil, 5th-8th May 2014).

Flipse, S.M., van der Sanden, M.C.A., Radstake, M., De Winde, J.H. and Osseweijer, P. (2014). 'The DNA of socially responsible innovation'. EMBO reports $15(2)$, pp. $134-137$.

Jones, R.A.L. (2011). 'Public engagement in and evolving science policy landscape'. In: Successful Science Communication. Telling It Like it is. Ed. by D.J. Bennett, D.J. and R.C. Jennings. Cambridge, U.K.: Cambridge University Press.

Kropp, K. and Blok, A. (2011). 'Mode-2 social science knowledge production? The case of Danish sociology between institutional crisis and new welfare stabilizations'. Science and Public Policy 38 (3), pp. 213-224.

Littlejohn, S.W. and Foss, K.A. (2008). Theories of human communication. Boston, U.S.A.: Wadsworth.

Luhmann, N. (1995). Social systems. Stanford, U.S.A.: Stanford University Press. Meyer, M. (2010). 'The rise of knowledge broker'. Sci. Commun. 32, pp. 118-127.

Mulder, H.A.J., Longnecker, N. and Davies (2008). 'The state of science communication programs at universities around the world'. Sci. Commun. 30 (2), pp. 277-287.

Nowotny, H.P., Scott, P. and Gibbons, M. (2001). Rethinking science: knowledge and the public in an age of uncertainty. Cambridge, U.K.: Polity Press.

Penders, B., Verbakel, J.M.A. and Nelis, A. (2009). 'The Social Study of Corporate Science: a research manifesto'. Bulletin of Science Technology $\mathcal{E}$ Society 29 (6), pp. 439-446.

Pennington, D.D. (2011). 'Bridging the disciplinary divide: co-creating research ideas in eScience teams'. Computer Supported Cooperative Work 20, pp. 165-196.

Sanden, M.C.A. van der and Meijman, F.J. (2007). 'Medische publiekscommunicatie: de contouren van systeemtheorie'. In: Basisboek wetenschapscommunicatie. Ed. by J. Willems. Amsterdam, The Netherlands: Boom.

Sawyer, R.K. (2005). Social emergence. Societies as complex systems. Cambridge, U.K.: Cambridge University Press.

Scheufele, D.A. (2014). 'Science communication as political communication'. PNAS 111 (4), pp. 13585-13592.

Schön, D. (1983). The reflective practitioner: how professionals think in action. New York, U.S.A.: Basic Books.

Steinmetz, G. (2007). 'The relations between sociology and history in the United States. The current state of affairs'. Journal of Historical Sociology 20 (1-2), pp. 1-12.

Stilgoe, J., Owen, R. and Macnaghten, P. (2013). 'Developing a framework for responsible innovation'. Research Policy 42, pp. 1568-1580.

Trench, B. (2008). 'Towards an analytical framework of science communication models'. In: Communicating Science in Social Contexts: New Models, New Practices. Ed. by D. Cheng, M. Claessens, T. Cascoigne, J. Metcalfe, B. Schiele and S. Shi. New York, Springer, U.S.A.: Springer. 
Tromp, N. (2013). 'Social design: how products and services can help us act in ways that benefit society'. Thesis. Delft University of Technology, The Netherlands.

van der Sanden, M.C.A. and Meijman, F.J. (2008). 'Dialogue guides awareness and understanding of science: an essay on different goals of dialogue leading to different science communication approaches'. Pub. Underst. Sci. 17 (1), pp. 89-103.

van der Sanden, M.C.A. and Osseweijer, P. (2011). 'Effectively embedding science communication in academia: a second paradigm shift'. In: Successful Science Communication. Telling It Like it is. Ed. by D.J. Bennett, D.J. and R.C. Jennings. Cambridge, U.K.: Cambridge University Press.

van der Sanden, M.C.A., van Dam, K.H., Stragier, J. and Kobus, C.B.A. (2013). 'Simulation based decision support for strategic communication and marketing management concerning the consumer introduction of smart energy systems'. Journal for Communication Studies 6 (11), pp. 75-104.

van Riel, C.B.M. and Fombrun, C.J. (2007). Essentials of corporate communication: implementing practices for effective reputation management. New York, U.S.A.: Routledge.

Verouden, N. and van der Sanden, M.C.A. (2013). 'Strategic Communication in a Knowledge-Intensive Network: an Ethnographic study'. In: Conference on Corporate Communication 2013. (New York, U.S.A. June 2013). Ed. by ICC.

Wenger, E. (2000). 'Communities of practice and social learning systems'. Organization 7 (2), pp. 225-246.

Wynne, B. (2001). 'Creating public alienation: expert cultures of risks and ethics on GMOs'. Science as Culture 10, pp. 445-481.

Maarten C.A. van der Sanden, PhD. is an assistant professor of science communication at the Delft University of Technology, The Netherlands. He specializes in the social design of science communication processes and its supporting tools for scientists and science communication practitioners, from a social systems perspective. He teaches social systems and design courses in both MSc and BSc programs and is a member of communities of practice in science communication and corporate communication.

E-mail: m.c.a.vandersanden@tudelft.nl.

Steven M. Flipse, PhD. is an assistant professor of science communication at the Delft University of Technology, The Netherlands. He specializes in science communication and innovation from a responsible research and innovation perspective. He develops and designs supportive tools for scientists and science communication practitioners involved in innovation processes. He teaches science marketing and innovation classes in both MSc, BSc and Postgraduate programs. E-mail: s.m.flipse@tudelft.nl.

How to cite

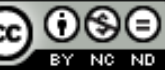

van der Sanden, M.C.A. and Flipse, S.M. (2015). 'A cybernetic dream: how a crisis in social sciences leads us to a Communication for Innovation-Laboratory'. JCOM 14 (01), Y01.

This article is licensed under the terms of the Creative Commons Attribution - NonCommercial NoDerivativeWorks 4.0 License.

ISSN 1824 - 2049. Published by SISSA Medialab. http:/ /jcom.sissa.it/. 